\title{
Anthropomorphic Differences Between the Distal Femora of Men and Women
}

\author{
Jess H. Lonner MD, Jeff G. Jasko MS, \\ Beverly S. Thomas RN
}

Published online: 22 August 2008

(c) The Association of Bone and Joint Surgeons 2008

\begin{abstract}
There is debate about whether distinct designs of femoral components for men and women are needed based on morphologic and size differences between genders. We asked whether anthropomorphic differences exist between the distal femoral dimensions in women and men. We measured the distal femora of 100 women and 100 men intraoperatively after preparation for prosthetic implantation. The measured dimensions included the anteroposterior height from the posterior edge of the medial femoral condyle to the flush anterior cut, the mediolateral width at the transepicondylar axis, the anterior and posterior edges of the anterior chamfer, and the medial and lateral trochlear flanges. These measurements were compared between genders using independent-samples $t$ test. The aspect ratio (a measure of the shape of the distal femur), the ratio between the anteroposterior and mediolateral dimensions, was calculated for men and women to determine whether there is a shape difference between genders. The mean aspect ratio was larger for women than for men $(0.84$ [range, 0.57-1.03] versus 0.81 [range, 0.066-1.34], respectively). The standard deviation and range of each measurement of size and morphology suggest variability
\end{abstract}

One of the authors (JHL) is a consultant for Zimmer, Inc, Warsaw, IN, and MAKO Surgical Corp, Fort Lauderdale, FL.

Each author certifies that his or her institution has approved the reporting of these cases, that all investigations were conducted in conformity with ethical principles of research, and that informed consent for participation in the study was obtained.

J. H. Lonner ( $\square)$, J. G. Jasko, B. S. Thomas

Booth Bartolozzi Balderston Orthopaedics, Pennsylvania

Hospital, 800 Spruce Street, Philadelphia,

PA 19107, USA

e-mail: lonnerj@pahosp.com not only between genders but also within genders. Whether the aspect ratios and variations will reflect clinically important differences in outcomes after TKA with available prostheses will require additional study.

\section{Introduction}

There has been considerable recent debate regarding whether distinct TKA components with different sizes and shapes are necessary to accommodate morphologic differences of the distal femur between men and women $[2,4,6$, $7,9,11,12]$. While some studies have suggested there may be increased pain in female patients after TKA, it is not clear whether these differences are related to implant design or other factors $[6,12]$. Given women are the recipients of approximately $2 / 3$ of all TKAs performed [5], if in fact there are morphologic differences in the shape and dimensions of the distal femur that account for the disparities in functional and clinical results after TKA based on gender (or ethnicity, race, or other factors, for that matter), then a design that accommodates these differences might be appropriate. There is an evolving interest in having accessible a greater variety of sizing and shape options for patients during TKA to more accurately address even subtle morphologic variability among people.

Chin et al. [3] studied the anteroposterior (AP) and mediolateral (ML) dimensions of 200 consecutive osteoarthritic knees undergoing unilateral primary TKA and found, on average, the distal femoral height was $10.5 \%$ taller in men than in women and the mean ML dimension was $13.7 \%$ wider in men than in women, with an aspect ratio (AP/ML ratio) of 0.82 for women and 0.79 for men. This difference indicates not only that men on average have larger distal femora than women but the shapes are 
different [3]. Similar anthropometric gender differences were reported in a study by Hitt et al. [7], which suggested disparities account for femoral component overhang in women and underhang in men with several contemporary implants.

The questions we sought to answer are whether there are gender differences in the dimensions and morphology of the distal femora between men and women and whether the calculated aspect ratios corroborate the findings of previous studies.

\section{Materials and Methods}

We collected geometric data from the distal femora of 100 consecutive men and 100 consecutive women undergoing unilateral primary TKA for osteoarthritis. We excluded 12 patients who had posttraumatic arthritis with intraarticular fracture and two with severe bony deficiency from rheumatoid arthritis that would render measurements inaccurate. The mean patient age was 67 years in men (range, 45-83 years) and 68 years in women (range, 48-88 years). Right knees were studied in $62 \%$ of men and $56 \%$ of women. The mean height of men in the study was 69.3 inches (range, 56-78 inches); the mean height of women was 62.5 inches (range, 52-71 inches). The mean body mass index was 32 (range, 21-51) for men and 34 (range, 20-58) for women.

All surgeries and all measurements were performed by the senior author (JHL). Each measurement was made after resecting the distal femur, anterior chamfer, and anterior trochlea. Anterior referencing instrumentation was used to resect the trochlea, ensuring the cut was flush with the anterior femoral cortex. There were no cases with anterior notching. Additionally, all medial and lateral osteophytes were removed before measuring the distal femur. The initial distal femoral resection removed $7 \mathrm{~mm}$ of bone from the more prominent distal condylar surface (usually the medial femoral condyle), at a valgus angle ranging from $4^{\circ}$ to $6^{\circ}$. A sterile caliper was used to make all measurements, which were recorded intraoperatively. Measurements included the AP dimension of the distal femur from the flush anterior femoral resection to the unresected surface of the posterior medial femoral condyle, the ML dimension at the level of the transepicondylar axis, the ML width at the anterior edge of the anterior chamfer, the ML width at the posterior edge of the anterior chamfer, the height of the lateral trochlea, and the height of the medial trochlea (see Appendix 1).

The ratio of the AP to ML (at the level of the transepicondylar axis) dimensions, the so-called aspect ratio, was calculated. We compared mean measurements between genders using the Mann-Whitney $U$ test. This nonparametric method was employed because the Shapiro-Wilk test for normality revealed the distributions of the measurements were generally asymmetric.

\section{Results}

The mean aspect ratio was larger for women than for men (0.84 [range, 0.57-1.03] versus 0.81 [range, 0.066-1.34], respectively) (Table 1). Seventy-six percent of women had an ML dimension at the level of the transepicondylar axis

Table 1. Dimensions of the distal femora in male and female patients

\begin{tabular}{|c|c|c|c|c|c|}
\hline Dimension & Gender & Average & Minimum & Maximum & $\mathrm{p}$ value \\
\hline \multirow[t]{2}{*}{ Ratio (AP/ML) } & Female & 0.84 & 0.57 & 1.03 & \multirow[t]{2}{*}{0.001} \\
\hline & Male & 0.81 & 0.66 & 1.34 & \\
\hline \multirow[t]{2}{*}{$\mathrm{AP}(\mathrm{mm})$} & Female & 56.32 & 44.0 & 70.0 & \multirow[t]{2}{*}{$<0.001$} \\
\hline & Male & 62.27 & 52.5 & 74.0 & \\
\hline \multirow[t]{2}{*}{ ML at TEA $(\mathrm{mm})$} & Female & 67.49 & 57.5 & 87.0 & \multirow[t]{2}{*}{$<0.001$} \\
\hline & Male & 76.92 & 45.0 & 89.0 & \\
\hline \multirow[t]{2}{*}{ Anterior edge of chamfer (mm) } & Female & 48.93 & 40.0 & 60.0 & \multirow[t]{2}{*}{$<0.001$} \\
\hline & Male & 56.29 & 43.0 & 68.5 & \\
\hline \multirow[t]{2}{*}{ Posterior edge of chamfer $(\mathrm{mm})$} & Female & 57.42 & 49.5 & 67.0 & \multirow[t]{2}{*}{$<0.001$} \\
\hline & Male & 67.74 & 56.0 & 78.0 & \\
\hline \multirow[t]{2}{*}{ Lateral trochlea $(\mathrm{mm})$} & Female & 10.47 & 2.0 & 19.0 & \multirow[t]{2}{*}{$<0.001$} \\
\hline & Male & 11.95 & 6.5 & 20.5 & \\
\hline \multirow[t]{2}{*}{ Medial trochlea $(\mathrm{mm})$} & Female & 6.95 & 2.0 & 18.0 & \multirow[t]{2}{*}{0.030} \\
\hline & Male & 7.71 & 1.0 & 18.0 & \\
\hline
\end{tabular}

$\mathrm{TEA}=$ transepicondylar axis; $\mathrm{AP}=$ anteroposterior; $\mathrm{ML}=$ mediolateral. 


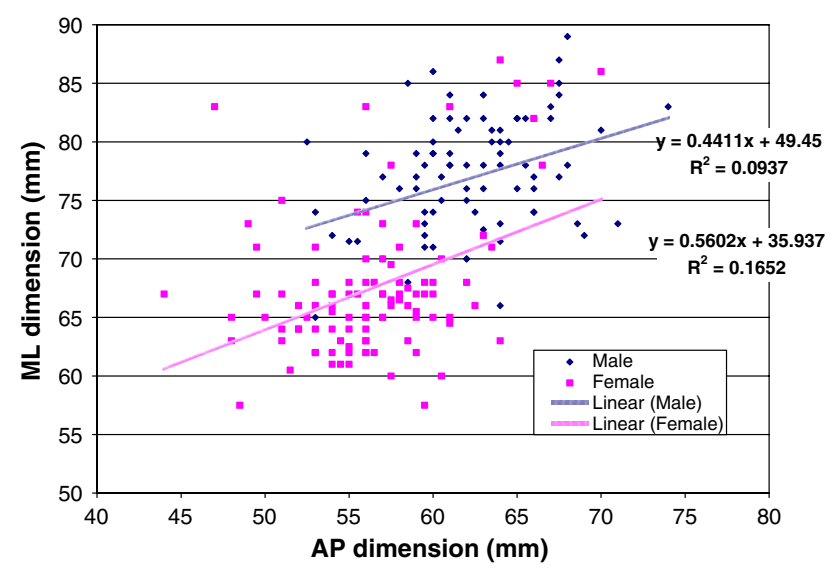

Fig. 1 A graphic depicts the ratio of AP and ML dimensions of male and female distal femora.

between or equal to $60 \mathrm{~mm}$ and $70 \mathrm{~mm}$, compared to $8 \%$ of men; $22 \%$ were greater than $70 \mathrm{~mm}$, compared to $89 \%$ of men; and $2 \%$ were less than $60 \mathrm{~mm}$, compared to $1 \%$ of men. Nineteen percent of women had an AP dimension greater than or equal to $60 \mathrm{~mm}$, compared to $85 \%$ of men; $50 \%$ had an AP dimension between 55 and $60 \mathrm{~mm}$, compared to $3.5 \%$ of men; $31 \%$ of women had an AP dimension less than $55 \mathrm{~mm}$, compared to $0 \%$ of men. Five percent of the women had an AP measurement less than $50 \mathrm{~mm}$ (Fig. 1). There was an insignificant trend toward larger aspect ratios in both men and women in the top $10 \%$ for height compared to the middle $80 \%$ and the shortest $10 \%$. The mean aspect ratios for the shortest $10 \%$ of both men and women were insignificantly smaller than in the remaining $90 \%$ with the same gender. While there was variation in aspect ratio within genders, there was little variation according to height or body mass index.

\section{Discussion}

There are conflicting suggestions as to whether there is a need for distinct designs of femoral components for men and women based on morphologic and size differences between genders. We sought to answer whether anthropomorphic differences exist between the distal femoral dimensions in women and men by measuring the distal femora of 100 women and 100 men intraoperatively after 7 -mm resection of the distal femur in approximately $4^{\circ}$ to $6^{\circ}$ of valgus using an intramedullary cutting guide.

We found evidence of sexual dimorphism when comparing men and women, not only in the dimensions of the distal femur, but also in the morphology and shape, as represented by the aspect ratio. While neither a formal power analysis nor a subanalysis based on ethnicity or race was performed, we believe the consecutive nature of sampling is representative of the typical patients that are seen in a metropolitan mid-Atlantic tertiary practice. We did not perform an a priori power analysis to identify the appropriate number of knees to study in each gender since it would have required a targeted difference in mean measurements between genders; we do not know what difference in aspect ratios are clinically important. However, if we presume the AP or ML dimensions range from 44 to $89 \mathrm{~mm}$ (Table 1), then a difference in the mean aspect ratios between the genders of 0.03 ( 0.84 for females and 0.81 for males) would essentially reflect variations of 1.3 $2.7 \mathrm{~mm}$. Further, it remains unproven in clinical series what difference in aspect ratio between the femoral component and native femur may have a clinically important impact on functional and clinical outcomes. We did not seek to answer that question and it will require further study.

Measuring the distal femur after distal femoral and anterior resection was considered a reasonable approach because it is easier to rest calipers on the cut surfaces and minimize tilting of the calipers that would potentially occur on an uncut distal femur. The choice to measure the femoral geometry after a $7-\mathrm{mm}$ resection is appropriate, given it is often the point of resection on which the femoral component lies. While changes in varus/valgus obliquity of the resection may alter the ML dimensions of the femur, it is clinically appropriate, as the measurements were made in 200 femora prepared by one surgeon before femoral implantation. Trochlear resections were made flush with the anterior femoral surface. While no cases were associated with notching of the anterior femoral cortex or an anteriorized cut, small errors in trochlear resection could alter the measurements and calculations.

Anthropomorphic differences in long bone geometry have been used in the area of forensics to determine gender in skeletal remains and specimens [8, 13]. Others have reported variation in AP and ML dimensions when comparing the Indian and Western populations and discussed the implications of these differences in implant design. In the study by Vaidya et al. [14], available implants were adequate to accommodate the AP and ML dimensions of the femur in $86.8 \%$ of Indian men but in only $40 \%$ of women. Sixty percent of Indian women studied had femoral AP dimensions smaller than the smallest available implant. Additionally, among Indian patients, the ML dimensions varied by more than $10 \mathrm{~mm}$ for a constant AP size, suggesting a need for two ML dimensions for each AP size of implant [14]. Our study did not address whether there are morphologic differences in the distal femur based on race or ethnicity. It only sought to address whether there are shape differences based on gender. A study by Poilvach et al. [11] reported a difference between male and female patients in the ratio between the transepicondylar width and 
the mean height of the femoral condyles. In that series, the average aspect ratio was 0.75 for men and 0.77 for women, suggesting a need for use of narrower femoral components in female patients to avoid ML overhang of prosthetic components during TKA [11]. A study by Chin et al. [3] reported the mean aspect ratio for all patients was 0.8 , with a difference between women and men $(0.82$ and 0.79 , respectively). None of the cited studies or the present one sought to address what the clinical implications of the variations in aspect ratio are in terms of functional outcomes, pain, and range of motion.

Recognizing the femora of women are typically narrower distally than the femora of men, there is occasionally a need to downsize the femoral components in women during TKA, which either results in notching anteriorly or additional resection of the posterior femoral condyles. Notching can predispose to periprosthetic fractures. The more typical solution, namely additional posterior condylar resection, results in an imbalance between the flexion and extension gaps. That is addressed by resection of additional distal femur, which balances the flexion and extension spaces but elevates the joint line. That process may have subtle kinematic implications that can affect the performance of knees and perhaps long-term outcomes after TKA. It may also account for some of the gender differences observed in some studies with regard to outcomes.

Chin et al. [3] suggested a prosthetic knee with a constant AP/ML ratio of 0.8 among various sizes may provide the most optimal fit across genders. However, an alternate approach would be to provide implants with different aspect ratios to accommodate morphologic differences among people, whether or not it has to do with gender. In our practice, nearly $5 \%$ of men have been recipients of a TKA designed to accommodate the femoral morphology more typical in women. The aspect ratio differences between genders often result in femoral component overhang in women and underhang in men [7]. Medial or lateral overhang can cause soft tissue irritation or compromise soft tissue tension of the extensor mechanism. It may also account for a tendency for anterior knee pain or loss of flexibility in some patients after TKA. Therefore, having several component shapes available may be appropriate if additional study shows that necessary.

We also found a greater thickness of the medial and lateral trochlear dimensions in men compared to women. This corroborates the findings of Hitt et al. [7]. The implications of an overstuffed patellofemoral compartment from either a patellar composite thickness that is excessive or trochlear stuffing are not well understood, although a study by Bengs and Scott [1] reported, under anesthesia, each $2-\mathrm{mm}$ incremental increase in patellar thickness resulted in a $3^{\circ}$ decrease in passive knee flexion after TKA. Overstuffing on the trochlear side can have a potential detrimental effect as well. In a cadaver study by Mihalko et al. [10], each 2-mm incremental increase of the trochlear flange thickness was associated with a $2^{\circ}$ loss of passive knee flexion. Given the patella thickness in women on average is $3 \mathrm{~mm}$ thinner than it is in men [4], the risk of overstuffing the patellofemoral compartment in women from either an underresected patella or relatively thick patellar or trochlear prosthesis surfaces may ultimately contribute to some of the patellofemoral problems observed in women after TKA, such as pain or loss of motion. Our study did not address this issue.

The anthropomorphic differences between the distal femora in men and women support the argument that there may be value to having available a greater range of implants to accommodate dimensional differences between the genders and possibly other morphologic differences around the knee. The standard deviation of each measurement of size and morphology we observed suggests variability not only between genders but also within genders. The distal femoral shapes of large women did not tend to resemble femora of men and the distal femora of small men did not tend to resemble morphologically the distal femora of women. Further study will be necessary to see whether gender- or ethnic-based modifications in implant design will influence clinical outcomes in TKA recipients.

Acknowledgments We thank Mr. Jordan Rosenblatt for technical assistance. 


\section{Appendix 1}

Diagram of Measurements

MRN = medical record number; Lat troch $=$ lateral trochlea; Med troch $=$ medial trochlea; $\mathrm{AP}=$ anteroposterior; $\mathrm{ML}=$ mediolateral; $\mathrm{Lat}=$ lateral; $\mathrm{Med}=$ medial; $\mathrm{DJD}=$ degenerative joint disease; $\mathrm{AVN}=$ avascular necrosis; $\mathrm{RA}=$ rheumatoid arthritis

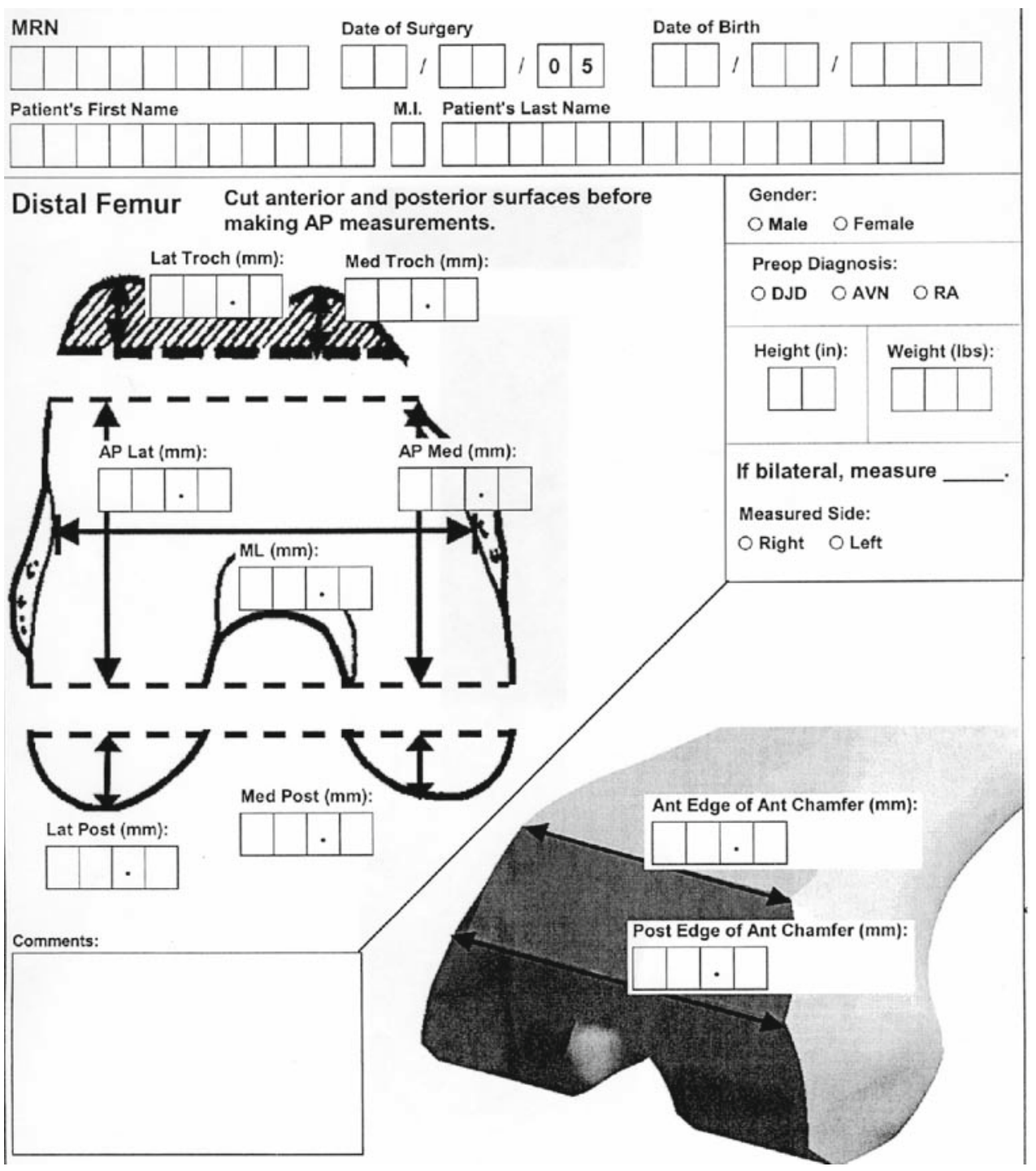

\section{References}

1. Bengs BC, Scott RD. The effect of patellar thickness on intraoperative knee flexion and patellar tracking in total knee arthroplasty. J Arthroplasty. 2006;21:650-655.

2. Booth RE. Sex and the total knee: gender-sensitive designs. Orthopedics. 2006;29:836-838.

3. Chin KR, Dalury DF, Zurakowski D, Scott RD. Intraoperative measurements of male and female distal femurs during primary total knee arthroplasty. J Knee Surg. 2002;15:213-217.

4. Conley S, Rosenberg A, Crowninshield R. The female knee: anatomic variations. J Am Acad Orthop Surg. 2007;15(Suppl 1):S31-S36
5. DeFrances CJ, Cullen KA, Kozack LJ. National Hospital Discharge Survey: 2005 annual summary with detailed diagnosis and procedure data. Vital Health Stat 13. 2007;165:1-209.

6. Fisher DA, Dierckman B, Watts M. Looks good but feels bad: factors that contribute to poor results after total knee arthroplasty. J Arthrop. 2007;22(6 Suppl 2):39-42.

7. Hitt K, Shurman JR, Greene K, McCarthy J, Moskal J, Hoeman T, Mont MA. Anthropometric measurements of the human knee: correlation to the sizing of current knee arthroplasty systems. J Bone Joint Surg Am. 2003;85:115-122.

8. King CA, Iscan MY, Loth SR. Metric and comparative analysis of sexual dimorphism in the Thai femur. J Forensic Sci. 1998;43:954-958. 
9. MacDonald SJ, Charron KD, Bourne RB, Naudie DD, McCalden $\mathrm{RW}$, Rorabeck $\mathrm{CH}$. The John Insall Award: Gender-specific total knee replacement: prospectively collected clinical outcomes. Clin Orthop Relat Res. 2008;466. Doi:10.1007/s11999-008-0430-1.

10. Mihalko W, Fishkin Z, Krackow K. Patellofemoral overstuff and its relationship to flexion after total knee arthroplasty. Clin Orthop Relat Res. 2006;449:283-287.

11. Poilvach PL, Insall JN, Scuderi GR, Font-Rodriguez DE. Rotational landmarks and sizing of the distal femur in total knee arthroplasty. Clin Orthop Relat Res. 1996;331:35-46.
12. Singh JA, Gabriel S, Lewallen D. The impact of gender, age, and preoperative pain severity on pain after TKA. Clin Orthop Relat Res. 2008;466. Doi:10.1007/s11999-0080399-9.

13. Steyn M, Iscan MY. Sex determination from the femur and tibia in South African Whites. Forensic Sci Int. 1997;90: 111-119.

14. Vaidya SV, Ranawat CS, Aroojis A, Laud NS. Anthropometric measurements to design total knee prosthesis for the Indian population. J Arthroplasty. 2000;15:79-85. 